Methods Urine samples from men, 10 patients with urethritis and $>5$ $\mathrm{PMNL} / \mathrm{hpf}$ and 10 healthy controls with $<5 \mathrm{PMNL} / \mathrm{hpf}$, were collected. All samples were tested for Neisseria gonorrhoeae, Chlamydia trachomatis, Mycoplasma genitalium, Ureaplasma urealyticum, U parvum, Trichomonas vaginalis, Herpes Simplex Virus type 1 and 2 and Adenovirus using specific PCR assays. The V3 and V4 regions of the $16 \mathrm{~S}$ rRNA gene were PCR amplified, tagged and sequenced using the Titanium kit and GS FLX pyrose-quencing system (Roche) according to manufacturer's instructions. Sequences were analysed using the RDP Pyrosequencing Pipeline and CLC Genomics Workbench.

Results From each of the 20 samples, 8150 quality filtered sequences were randomly selected. Sequences were assigned to the genus level using the RDP Classifier. A total of 172 gen-era were identified, 133 in patients and 104 in controls. The median number of genera was 35.5 (19-49) in patients with urethritis compared to $25(16-43)$ in the controls. No single genus was present in all samples. Members of the genera Pseudomonas and Sphingomonas were present in all the controls and in most of the patient samples. Also, representatives of the genera Brevundimonas, Micrococcus, Bradyrhizobium and Chryseobacterium were present in 15-18 of the samples. Of the 172 genera, 77 were only found in a single sample and 33 were found in two different samples. Rarefaction analysis at the $5 \%$ level (comparable to genus) suggested that all the controls and most urethritis samples harboured each between 31 and 125 different groups. Four of the urethritis samples apparently contained between 150 and 300 different groups.

Conclusions Urine, even from healthy men, contains a very diverse micro-flora. Though not statistically significant, the total and median number of genera was found higher in patients with urethritis than in controls. Several widespread genera are likely to represent commensals and bacteria present in the environment.

\section{P4-S4.02 A 22-ORGANISM MICROARRAY APPROACH FOR DETECTING MICROBIOLOGICAL ASSOCIATIONS WITH SYMPTOMATIC URETHRITIS IN MALES}

doi:10.1136/sextrans-2011-050108.527

${ }^{1} S$ Patel, ${ }^{2} \mathrm{M}$ Pond. ${ }^{1}$ St George's Healthcare NHS Trust, London, UK; ${ }^{2}$ St George's, University of London, London, UK

Background In addition to its known microbiological aetiology, urethritis in men may be linked with other genital tract organisms, as yet unidentified in its pathogenesis. We used a microarray, with capacity to detect 22 genital tract organisms, in order to determine the association of symptomatic urethritis with infection or carriage of these organisms.

Methods 129 patients were asked to provide an extra first void urine specimen or give permission for their residual urine specimen submitted for Chlamydia trachomatis NAAT testing to be utilised. Patients were categorised into three self-reported symptom groups: definite symptoms of urethritis (discharge and/or dysuria), category 1 (C1) $n=80$; non-specific symptoms of urethritis (eg, minimal urethral discomfort), category 2 (C2) $n=26$; and asymptomatic category 3 (C3) $n=23$. Total urine nucleic acid was extracted and subsequently used for PCR coupled microarray analysis. Organisms were defined as present or absent using an online data analysis method. In a pre-planned analysis, the following categories were compared for prevalence of organisms: C1 vs C2 and C3 combined; C1 and C2 combined vs C3 using Fisher's exact test.

Results One or more organisms were detected in $74 \%(n=95)$ of patients and two or more organisms in $33 \%(n=42)$. The prevalence of organisms known to cause urethritis in this largely symptomatic cohort was: $16 \%(n=21), 9 \%(n=12)$ and $5 \%(n=6)$ for $C$ trachomatis, Mycoplasma genitalium and for Neisseria gonorrhoeae respectively. Escherichia coli was the most prevalent organism detected with a prevalence of $18 \%(n=23)$. The presence of $M$ genitalium was stat- istically associated with $\mathrm{C} 1$ and $\mathrm{C} 1$ and $\mathrm{C} 2$ combined ( $\mathrm{p}=0.03$ and 0.01 respectively). In symptomatic patients, $C$ trachomatis, Ureaplasma urealyticum, and Gardnerella vaginalis appeared to be more prevalent than in asymptomatics although not statistically significantly. Lactobacilli where detected in $1.3 \%$ and $4 \%$ of patients with C1 and C2 symptoms respectively, compared with $17 \%$ of asymptomatic patients. The absence of lactobacilli was associated with urethritis symptoms, either C1 alone or C1 and C2 combined $(p=0.03$ and $p=0.01$ ) respectively.

Conclusions Using a polymicrobial microarray approach we have demonstrated that symptomatic urethritis is associated with depletion of lactobacilli. This confirms early work using urethral swabs. The temporal nature of Lactobacilli depletion in relation to the onset of symptomatic urethritis needs to be investigated further.

\section{P4-S4.03 A LOW-COST MICROFLUIDICS-BASED DIAGNOSTIC TEST FOR STDs}

doi:10.1136/sextrans-2011-050108.528

\section{S Sia. Columbia University, New York, USA}

Background Undiagnosed and untreated STIs cause large morbidity and mortality, including birth defects and stillborn babies. Since most STI's have known treatments, the largest barriers for treating patients include high cost of transporting specimens to central labs and lack of access to diagnostic testing. We present data on a portable and low-cost microfluidics device for point-of-care diagnosis of multiple STDs (such as HIV and syphilis) in combination. In order to reduce the cost and size of the assay while maintaining high performance, we incorporated microfluidic designs such as single-use plastic microfluidic cassettes, a passive method for delivering reagents, and an amplification chemistry using gold nanoparticles.

Methods Our overall device is named mChip (mobile microfluidic chip for immunoassay on protein markers). We demonstrated an ability of mChip to simultaneously detect antibodies against HIV and syphilis in needle-pricked sample volumes. Both commercial specimens and archived specimens from Sub-Saharan Africa were used.

Results The test sensitivities and specificities for detection of HIVspecific antibodies and treponemal-specific antibodies matched the performances from lab-based ELISA. Compared to ELISA, our test can be performed anywhere, uses a very small volume of blood, and is about 10 times faster. Some of these results are in press in Nature Medicine, 2010.

Conclusion The mChip provided excellent performance in the diagnosis of HIV using only $1 \mu \mathrm{l}$ of unprocessed whole-blood and $<15$ min assay-time, and an ability to simultaneously diagnose HIV and syphilis with sensitivities and specificities equal to lab-based assays. Overall, we demonstrate an integrated strategy for miniaturising complex laboratory assays using microfluidics and nanoparticles to enable POC diagnostics and early detection of infectious diseases in remote settings.

\section{P4-S4.04 TEMPORAL DYNAMICS OF VAGINAL BACTERIAL COMMUNITIES}

doi:10.1136/sextrans-2011-050108.529

1J Ravel, 'R Brotman, ${ }^{1} \mathrm{P}$ Gajer, ${ }^{1} \mathrm{~J}$ Sakamoto, ${ }^{1} \mathrm{~S}$ Koenig, ${ }^{1} \mathrm{~L}$ Fu, ${ }^{2} \mathrm{X}$ Zhou, ${ }^{2} \mathrm{Z}$ Abdo, ${ }^{2} \mathrm{~L}$ Forney, 'J Ravel. 'University of Maryland, School of Medicine, Baltimore, USA; ${ }^{2}$ University of Idaho, USA

Background Dysbiosis of vaginal bacterial communities have been associated with increased risk for sexually transmitted infections and bacterial vaginosis. This is the first observational study to model temporal dynamics of vaginal microbiota using frequently collected samples, behavioural data and culture-independent methods. 
Methods Thirty-three asymptomatic, reproductive-age women selfcollected mid-vaginal swabs every 3rd day for 16 weeks (998 samples). Participants reported behaviours and menstrual data on daily diaries. Bacterial communities were determined by pyrosequencing of barcoded 16S rRNA genes (V1-V2 region). Participants were clustered into five community classes based on temporal patterns of vaginal bacterial community composition using transition probabilities. A linear mixed effect model for the log of Jensen-Shannon rate of community change was utilised. The model accounted for correlations between samples from the same participant and was adjusted for time-varying confounders and normalised menstrual cycle time.

Results Three of the community classes were most often dominated by Lactobacillus iners, $L$ crispatus, or $L$ gasseri, respectively, while two lacked significant numbers of Lactobacillus spp. The latter classes were split into subtype A typified by Corynebacterium, Anaerococcus, Peptinophilus, Prevotella, and Finegoldia, while those of subtype B showed a higher abundance of the genus Atopobium. The rank abundance and species composition of bacterial communities in some women changed markedly over short periods of time while others were relatively stable. Classes dominated by $L$ crispatus and $L$ gasseri experienced the fewest fluctuations in community composition, and communities that lacked significant number of Lactobacillus spp. also demonstrated some stability. Vaginal communities dominated by $L$ iners demonstrated either a lack of constancy or notable stability. The menstrual cycle was associated with temporal dynamics, but these effects were influenced by bacterial community class. Sexual activity the day prior to sampling was of borderline statistical significance $(p=0.065)$ and is a variable of interest in supplementary modelling. Conclusions Vaginal microbiota can fluctuate rapidly. Future studies should investigate the role of temporal changes in vaginal microbiota on sexually transmitted infection risk. Longitudinal studies of the vaginal microbiome may allow for the future development of targeted individualised therapeutic approaches.

\section{Health services and policy poster session 1: Stigmatisation and Mental Health \\ P5-S1.01 PERCEPTIONS AND PRACTICES OF EMPLOYERS OF LABOUR IN IBADAN NORTH LOCAL GOVERNMIENT AREA TOWARDS PERSONS LIVING WITH HIV/AIDS}

doi:10.1136/sextrans-2011-050108.530

\section{Dipeolu, F Oshiname. University of Ibadan, Ibadan, Nigeria}

The epidemic of HIV and AIDS constitute one of the major challenges to development worldwide. The disease condition reduces the productivity of Persons Living with HIV and AIDS (PLWHA) in the labour force and imposes a huge socio-economic burden on employers of labour. In Nigeria, employers of labour's perceptions and practices relating to HIV and AIDS have not been adequately explored. This study therefore determined public and private employers of labour's perceptions, practices and attitudes to persons who are living with HIV and AIDS in Ibadan North Local Government Area, Oyo state, Nigeria. The study was cross-sectional in design. A multistage sampling technique was used to select 400 study participants in the public (38) and private (362) sectors for interview. The instrument for data collection was a pre-tested semistructured questionnaire. Data were analysed using descriptive statistics and the $\chi^{2}$ test. There were more males $(68.2 \%)$ than females (31.8\%) among the participants. A majority, (79.0\%), of the participants in the public sector (PuS) and $72.9 \%$ in the private sector (PrS) knew that an infected healthy looking person could harbour and transmit HIV to others. Overall, deep kissing (89.8\%) topped the list of perceived mode of transmission of HIV; blood transfusion was mentioned by $46.3 \%$ of the participants while unprotected sex $(30.4 \%)$ was the least mentioned. The listed ways of preventing HIV were: use of condom (85.9\%); avoiding deep kissing (71.3\%); keeping one uninfected sexual partner (21.2\%); and sexual abstinence $(15.4 \%)$. Avoidance of unscreened blood transfusion $(6.2 \%)$ was the least mentioned means of transmitting HIV. The perception of $77.0 \%$ of the entire participants was that HIV and AIDS do not reduce workers' productivity. A majority, (80.0\%), of which $2.3 \%$ with no formal education, $1.0 \%$ primary education, $13.5 \%$ secondary education, $41.5 \% \mathrm{HND} / \mathrm{B} . \mathrm{Sc}, 21.0 \%$ postgraduate and $0.8 \%$ with other qualifications were of the view that workers infected with HIV and AIDS should not be sacked. Slightly less than half $(48.0 \%)$ would keep their staff's HIV status secret while more than half, $(57.0 \%)$, would not recruit a PLWHA. More than half of the participants, (56.5\%), expressed a positive attitude to staff who is a PLWHA. More respondents in the private sector, (47.8\%), claimed to have ever organised HIV and AIDS-related educational programmes for their staff than those in the public sector $(42.1 \%)(p<0.05)$. Almost equal number of participants in the public $(36.8 \%)$ and private $(36.2 \%)$ sectors would require mandatory test for HIV before employment. Only $1.8 \%$ of participants in the $\mathrm{PuS}$ and $6 \%$ in the $\operatorname{PrS}(\mathrm{p}<0.05)$ reported that their organisations had a workplace HIV and AIDS policy. Although the participants would tolerate staff with HIV and AIDS, their perceptions are indicative of limited knowledge about the mode of transmission and prevention of HIV. Health education strategies such as training and workplace HIV and AIDS education are needed to address these shortcomings.

\section{P5-S1.02 SEXUAL RISK BEHAVIOURS AND MENTAL HEALTH CONCERNS AMONG HIV-INFECTED MSM}

doi:10.1136/sextrans-2011-050108.531

M Safran, T Guoyu, M Butler, K Hoover. Centers for Disease Control and Prevention (CDC), Atlanta, USA

Background Some HIV-infected MSM are at risk for acquisition and transmission of STDs because of ongoing high risk sexual behaviours. HIV-infected MSM also can experience psychosocial stressors and related mental health issues. We assessed the association between STD risk behaviours and the desire to discuss mental health issues, as reported by HIV-infected MSM currently receiving care in HIV clinics.

Methods In 2007, 426 HIV-infected MSM receiving care in eight urban HIV clinics were randomly selected to complete a survey that queried them about risk behaviours and health concerns. We estimated the percentages who answered that they wanted to discuss their mental health with their clinicians, by sexual behaviour and substance use in the past year. Differences in percentages of patients were considered statistically significant if the two tailed $p$ value was $\leq 0.05$ using a $\chi^{2}$ test.

Results $90 \%$ of patients had initiated care for their HIV infection more than a year ago. In the year preceding the survey, 74\% had multiple sexual partners, $75 \%$ engaged in anal intercourse, $48 \%$ had at least one HIV-uninfected partner, and $82 \%$ used illegal psychoactive drugs. Among those who reported anal intercourse, 39\% did not use a condom during the most recent episode. Among patients, $70 \%$ wanted to talk with their clinicians about how they felt mentally or emotionally. Patients who engaged in unprotected receptive anal sex were more likely to want such a conversation than those who did not engage in unprotected receptive anal sex ( $80 \%$ vs $62 \%, p<0.01$ ); those who engaged in unprotected insertive anal sex were also more likely to want a discussion ( $81 \%$ vs $63 \%$, $\mathrm{p}<0.01)$

Conclusions Although the vast majority of these patients had been in treatment for more than a year, a large percentage of patients 